\title{
Dyspnoea assessed by visual analogue scale in patients with chronic obstructive lung disease during progressive and high intensity exercise
}

\author{
André Noseda, Jean-Pierre Carpiaux, Jacqueline Schmerber, Jean-Claude Yernault
}

\begin{abstract}
Background A study was carried out to determine whether rating of dyspnoea by means of a visual analogue scale during a progressive exercise test is affected by the subject's awareness of the progressive nature of the protocol.

Methods Nineteen patients with chronic obstructive lung disease $\left(F E V_{1}\right.$ mean (SE) $1.06(0.07)$ 1) were studied. A preliminary incremental test was carried out with a work rate increasing by 10 watts every minute until the subject could no longer exercise, to determine the maximum work load (Wmax) and to anchor the upper end of the visual analogue scale. This was followed by two exercise tests performed one day apart in randomised sequence, with two different protocols. One was a 12 minute protocol that included two sudden bursts of three minute high intensity exercise, up to the subject's Wmax, each preceded by three minutes of low level exercise. The other test was a conventional three minute incremental test lasting 12 minutes. On both study days the only information given to the subject about the temporal profile of load was that a change would be made every three minutes. The relation between dyspnoea, as assessed by the visual analogue scale, and ventilation, measured during high intensity or progressive exercise, was studied.
\end{abstract}

Results The mean (SE) rates of increase of dyspnoea with increasing ventilation (\% of line length $1^{-1} \mathrm{~min}$ ) obtained by linear regression analysis were similar for the two tests $(2.86(0.20)$ for progressive exercise and $2.87(0.25)$ for high intensity exercise); it was $2.59(0.25)$ for the initial burst of high intensity exercise when the data on this were analysed separately. In six subjects with stable disease studied again two months later the reproducibility of the rating of dyspnoea was reasonably good for both protocols.

Conclusion The results suggest that in most patients with chronic obstructive lung disease the assessment of exercise induced dyspnoea by means of a visual analogue scale during a progressive exercise test is not affected by the subject's awareness of the progressive increase in work intensity.

Exercise induced dyspnoea is a major clinical problem, as it decreases the quality of life of many patients with chronic obstructive lung disease. Breathlessness can be assessed during exercise in the laboratory in normal subjects ${ }^{12}$ and in patients with chronic obstructive lung disease $^{34}$ by serial measurements with a visual analogue scale. Measurements of dyspnoea are usually analysed in relation to minute ventilation. Experimental evidence suggests that ventilation plays a part in the perception of breathlessness. ${ }^{5}$ The level of breathlessness associated with reflexly driven ventilation, however, has been shown to be substantially higher than that associated with voluntary overbreathing both in normal subjects ${ }^{6}$ and in patients with chronic obstructive lung disease. ${ }^{7}$ In normal subjects the relation between dyspnoea and ventilation may also be disturbed by a recent experience of loaded breathing, ${ }^{8}$ or even without external intervention, during steady state exercise. ${ }^{9}$ These findings show that perceived dyspnoea is a sensation different from the simple awareness of an increase in ventilation. When a visual analogue scale has been used to assess dyspnoea during exercise in patients with respiratory disease a progressive incremental test has usually been chosen. ${ }^{34}{ }^{10-13}$ Possibly, however, the rating of dyspnoea during such a test is biased by the progressive nature of the applied work load. The reproducibility of visual analogue scale scores in relation to exercise in patients with chronic obstructive lung disease has been examined only over short intervals. ${ }^{13}$

The principal aim of the present study was to investigate whether the assessment of dyspnoea in patients with chronic obstructive lung disease is affected by the subject's awareness of the progressive nature of the incremental protocol. As the use of purely randomised work loads is not practicable, we have designed an exercise protocol consisting of two sudden bursts of high intensity exercise, each preceded by a period of low level exercise. We have studied the relation between dyspnoea and ventilation during this high intensity exercise and during a conventional progressive exercise test in 19 outpatients with severe chronic obstructive lung disease, to assess whether the temporal profile of load influenced the relation between dyspnoea and ventilation. In addition, we have evaluated the reproducibility of the rating of exercise induced dyspnoea by these patients after a two month interval.

\section{Methods}

PATIENTS

Twenty one male outpatients with physician diagnosed chronic obstructive lung disease (history of cigarette smoking, chronic cough, 
and exertional dyspnoea) agreed to participate in a study on breathlessness. Their anthropometric characteristics were (mean (SE)): age 65 (1) years, height $172(1) \mathrm{cm}$, weight $71 \cdot 1$ $(2 \cdot 7) \mathrm{kg}$. All had an $\mathrm{FEV}_{1}$ below $55 \%$ predicted and $\mathrm{FEV}_{1} / \mathrm{FVC}$ below $55 \%$. All but one received regular oral or inhaled bronchodilators (or both); six of the 21 also inhaled corticosteroids. We excluded patients with appreciable reversibility of airflow limitation, defined as a $\triangle \mathrm{FEV}_{1}$ above $10 \%$ predicted after an inhaled bronchodilator. ${ }^{14}$ Subjects with known hypertension or heart disease, or unable for any reason to exercise maximally, were also excluded.

\section{STUDY DESIGN}

The study protocol was approved by the local ethics committee and all patients gave informed consent. The patients attended the hospital in the afternoon on three consecutive days and were studied in a stable period. No change in drug treatment was allowed. The subjects were asked to abstain if possible from inhaled bronchodilators four hours before attending the hospital. Each session included spirometry and a cycle exercise test. On the first day a progressive test was performed without ventilatory measurements to determine the subject's maximum work load and to anchor the upper end of the visual analogue scale used to assess breathlessness. On the second and third day two exercise protocols, including measurement of ventilation and assessment of dyspnoea, were performed in random order. One was a conventional progressive test and the other consisted of two high intensity exercise periods each preceded by a period of low level exercise. Six subjects were studied again two months later. They were carefully questioned to make sure that no change had occurred in exertional tolerance or in drug treatment. They attended in the afternoon on two consecutive days and the study design was the same except that no preliminary test was carried out.

\section{SPIROMETRY}

$\mathrm{FEV}_{1}$ was measured with a Microspiro HI-298 (Chest Corporation, Tokyo) spirometer. The reported $\mathrm{FEV}_{1}$ was the highest from three technically satisfactory manoeuvres, the two highest values varying by not more than $5 \%$. Predicted values were calculated on the basis of age and height. ${ }^{15}$

\section{VISUAL ANALOGUE SCALE}

The visual analogue scale was a $20 \mathrm{~cm}$ horizontal line ${ }^{1}$ with bipolar opposite descriptors"not at all breathless" (lower end 0\%) and "extremely breathless" (upper end 100\%). Each subject was instructed to mark the line at any point he wished to rate the severity of his breathlessness. Subjects were not reminded of their previous ratings. None of the subjects had experience of dyspnoea evaluation and all were unaware of the purpose of the experiment.
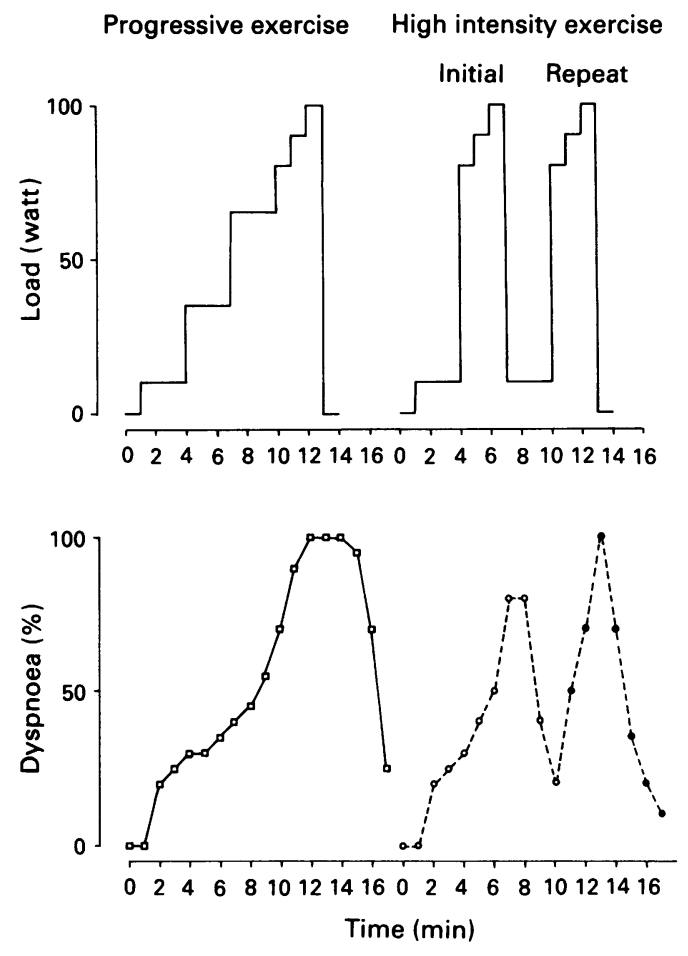

Figure 1 Work load and dyspnoea (\% of visual analogue scale length) during progressive and high intensity (initial and repeat) cycle exercise in a 58 year old patient with severe chronic obstructive lung disease (FEV $1 \cdot 24 l, 42 \%$ predicted).

\section{PRELIMINARY CYCLE TEST}

The subjects were tested in the exercise laboratory at least two hours after their last meal. Patients were instructed before the test to make a manual sign when they thought that they had nearly reached their maximum exercise tolerance. ${ }^{16}$ An Elema (Siemens, Erlangen, Germany) cycle ergometer was used. After unloaded pedalling during the first minute, the load was subsequently increased by 10 watts every minute. An electrocardiogram was used to measure heart rate and to monitor heart rhythm. At the time the manual sign was made verbal encouragement was given to urge the subject to maintain his effort for another 30 seconds or even one minute. The maximum work load (Wmax) was defined as the highest work level reached and maintained for a full minute. This value was used in the subsequent experiments to determine the protocols. At the moment the load was returned to zero watts the subject was shown the visual analogue scale and told that the intensity of his breathlessness at that moment was represented by the upper end of the scale $(100 \%)$. Subjects were questioned afterwards about the reason for stopping exercise; all confirmed that breathlessness was the limiting factor.

\section{EXERCISE PROTOCOLS}

The progressive exercise test included unloaded pedalling (one minute) and four three minute periods (fig 1). During the first three periods the work load was set respectively at 10 watts, $\mathrm{Wmax} / 3$, and $2 \times \mathrm{Wmax} / 3$ and then increased to $W \max -20 \mathrm{w}$ (first minute), to Wmax $-10 \mathrm{w}$ (second minute) and up to 


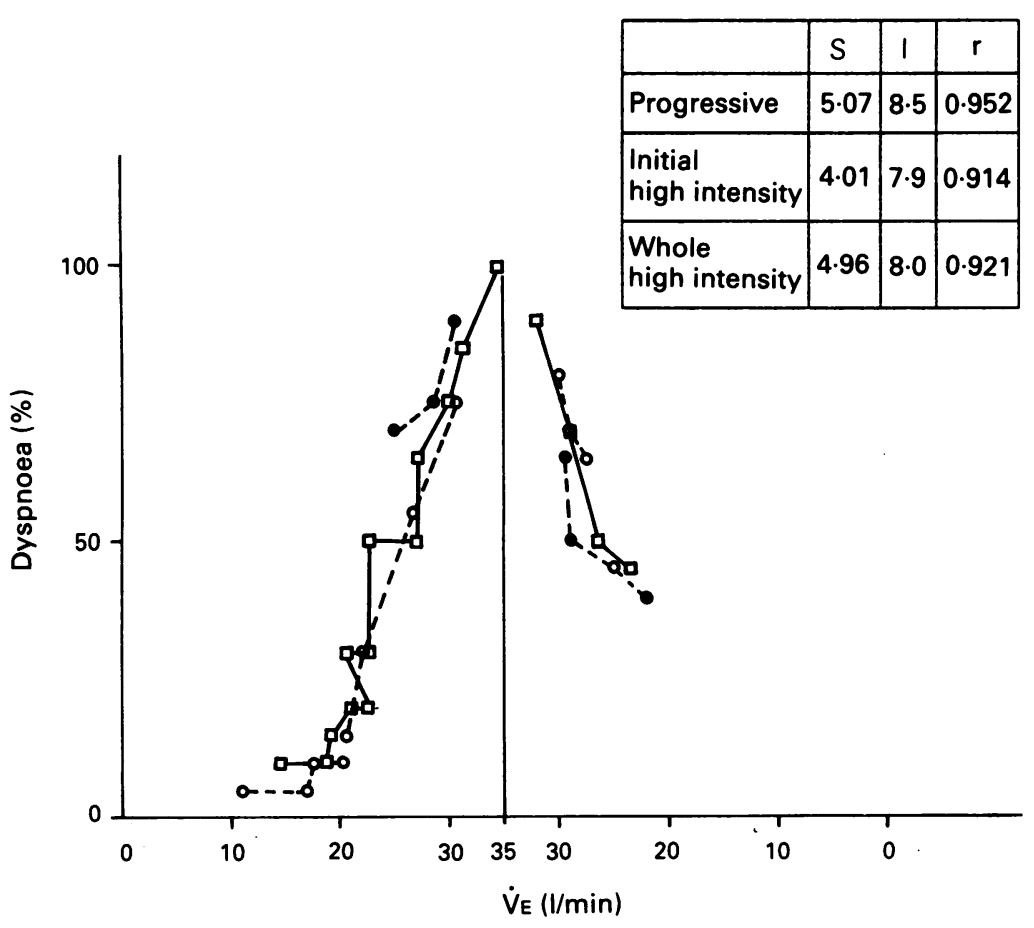

Figure 2 Dyspnoea/ventilation plot in a 71 year old patient with very severe chronic obstructive lung disease ( $F E V, 0.64 l, 23 \%$ predicted). Exercise of increasing intensity is on the left, recovery on the right. $\square-\square$ Progressive exercise; $\bigcirc----\bigcirc$ initial is on the left, recovery on the right. with the ventilation axis (I), and correlation coefficient ( $r$ ) obtained by linear regression are given for progressive and initial high intensity exercise and throughout high intensity exercise. This subject has the highest values for slope in the whole group.

Wmax (third minute) during the fourth period. Recovery included unloaded pedalling (one minute) followed by a three minute rest on the cycle. High intensity exercise included unloaded pedalling (one minute) and four three minute periods (fig 1). During the first and third periods the work load was set at 10 watts while the second and the fourth periods were high intensity phases at $W \max -20$ watts for the first minute, Wmax - 10 watts for the second minute, and $W \max$ for the third minute. These two periods are referred to as "initial high intensity" and "repeat high intensity" exercise. The recovery phase was the same as in the progressive exercise. Before each exercise test the subject was told that the work load would be changed every three minutes after the initial minute of unloaded pedalling. No additional information was given about how the load would be changed during the test.

CYCLE TESTS WITH ASSESSMENT OF DYSPNOEA

The equipment used was the same as for the

Table 1 Conventional indices and dyspnoea ratings: progressive versus high intensity exercise (mean (SE) values)

\begin{tabular}{lccc}
\hline & Progressive & Initial high intensity & Repeat high intensity \\
\hline HRmax (beats/min) & $135.6(2.0)^{\star \star}$ & $127.9(2.0)^{\star \star \dagger}$ & $136.5(2.7) \dagger$ \\
$\dot{V} \max (1 / \min )$ & $44.3(2.0)$ & $40.4(1 \cdot 8)$ & $42.3(1.9)$ \\
$\dot{V} o_{2} \max (\mathrm{ml} / \mathrm{min})$ & $1256(46) \dagger$ & $1129(35) \dagger$ & $1194(38)$ \\
$\operatorname{Dmax}(\%)$ & $89.3(2.9)$ & $84.6(3.4)^{\star}$ & $91.3(2.0) \star$
\end{tabular}

Significance of differences between protocols: ${ }^{\star} p<0.05 ;{ }^{\star \star} p<0.01 ;$; $<<0.001$.

HRmax-maximum heart rate; Vemax-maximum minute ventilation; Vo $\mathrm{O}_{2} \mathrm{max}-$ maximum oxygen consumption; Dmax-maximum dyspnoea (as percentage of visual analogue scale). preliminary cycle test day, with the addition of 30 second recordings of minute ventilation $(\dot{V} E)$, oxygen consumption, carbon dioxide production, and respiratory exchange ratio (Ergopneumotest Jaeger, Wurzburg, Germany). Before each test the pneumotachograph was calibrated with a calibrated syringe and gas analysers with known gases (Oxhydrique, Machelen, Belgium). Recordings were displayed on a computer. Maximum heart rate (HRmax), maximum oxygen uptake ( $\dot{\mathrm{VO}}_{2} \mathrm{max}$ ), and maximum ventilation (VEmax) were the highest values reached during progressive, initial, or repeat high intensity exercise. Dyspnoea was assessed on the visual analogue scale during the last 10 seconds of every one minute period, the first rating being obtained at rest, just before the start of cycling. Serial dyspnoea levels in one patient during both exercise protocols are shown in figure 1. Maximum dyspnoea (Dmax) was defined as the highest rating obtained during either progressive, initial, or repeat high intensity exercise.

\section{ANALYSIS}

Dyspnoea-ventilation plots were drawn for each subject (see fig 2) and the relation of dyspnoea to ventilation during exercise of increasing intensity was assessed by linear regression analysis. In each subject the linear regression was made on 14 values for progressive exercise, on eight values for initial high intensity exercise, and on 11 values for the total high intensity exercise. A separate analysis of the repeat high intensity exercise was not performed because the number of values was too small. The dyspnoea-ventilation slope (D/ $\dot{V}_{E}$ slope), the intercept with the ventilation axis, and the correlation coefficient were determined in each subject for progressive, initial high intensity, and total high intensity exercise.

The variability of the measurements made after two months was assessed from the mean group values and from within subject coefficients of variation.

\section{Results}

USE OF THE VISUAL ANALOGUE SCALE

Two subjects from the initial sample of 21 were judged not to understand the correct use of the visual analogue scale and were excluded. One was unable to rate decreasing breathlessness during recovery. The other was unable to anchor the upper end of the visual analogue scale on the preliminary day: on the next day he used only the lower end of the scale (Dmax $35 \%$ ) when extremely breathless. The results are for the 19 subjects who used the visual analogue scale correctly.

\section{SPIROMETRY AND MAXIMUM WORK LOAD} Mean (SE) $\mathrm{FEV}_{1}$ on the preliminary test day was $1.06(0.07) 1$ (range $0.49-1.561)$ or $35.3 \%$ $(2 \cdot 4 \%)$ predicted (range $17 \cdot 7-51 \cdot 4 \%)$. Values on the progressive exercise day $(1.01(0.07) 1)$ and on the high intensity exercise day $(1.02$ $(0.06) 1)$ were very similar. $W \max$ values in the preliminary incremental test varied, being 90 $(n=4), 100(n=6), 110(n=1), 120(n=1)$, 


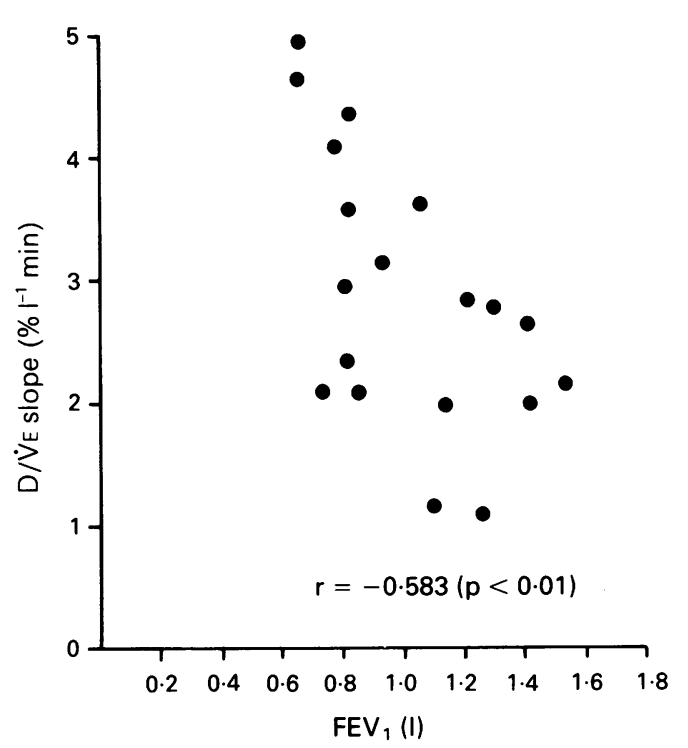

Figure 3 Dyspnoea/ventilation slope during high intensity exercise plotted against $F E V$, in 19 subjects with chronic obstructive lung disease.
Table 2 Relation of dyspnoea to ventilation during exercise of inceasing intensity: linear regression analysis (mean ( $S E$ ) values)

\begin{tabular}{lcll}
\hline & Progressive & $\begin{array}{l}\text { Initial high } \\
\text { intensity }\end{array}$ & $\begin{array}{l}\text { Whole high } \\
\text { intensity }\end{array}$ \\
\hline Slope of dyspnoea/ $/ \mathrm{V}$ plot $\left(\% 1^{-1} \mathrm{~min}\right)$ & $2 \cdot 86(0.20)$ & $2.59(0.25)$ & $2 \cdot 87(0.25)$ \\
Intercept with $\mathrm{VE}$ axis $(1 / \mathrm{min})$ & $10.0(1 \cdot 4)$ & $8.9(1 \cdot 1)$ & $9.5(1.3)$ \\
Correlation coefficient & $0.934(0.014)$ & $0.964(0.005)$ & $0.954(0.005)$ \\
\hline
\end{tabular}

$130(\mathrm{n}=5)$, and $140 \mathrm{w}(\mathrm{n}=2)$. Wmax and $\mathrm{FEV}_{1}$ on the preliminary test day were moderately well correlated (Wmax $=38.6 \times$ $\left.\mathrm{FEV}_{1}+70 \cdot 7 ; \mathrm{r}=0.64 ; \mathrm{p}<0.01\right)$.

PROGRESSIVE VERSUS HIGH INTENSITY EXERCISE: MAXIMUM VALUES

Maximum values of heart rate, ventilation, oxygen consumption, and dyspnoea associated with progressive, initial high intensity, and repeat high intensity exercise are shown in table 1. HRmax during initial high intensity exercise was significantly $(p<0.001)$ lower than during progressive and repeat high intensity exercise, and $\mathrm{V}_{2}$ max during initial high intensity exercise was lower $(p<0.001)$ than during progressive exercise. Dmax was slightly lower during initial than during repeat high intensity exercise $(p<0.05)$.

\section{DYSPNOEA-VENTILATION RELATIONSHIP}

Ten patients said that they were not breathless at all at rest on either study day; for the remainder the visual analogue scale rating at rest was (mean of both study days) $2.5 \%$ $(\mathrm{n}=1), 5 \%(\mathrm{n}=2), 7 \cdot 5 \%(\mathrm{n}=3), 10 \%$ $(n=2)$, and $20 \%(n=1)$. The results of linear regression analysis of the relation between visual analogue scale and $\dot{V} E$ are shown in table 2 and figure 3. For progressive exercise $r$ values exceeded 0.9 in 15 subjects (range $0.936-0.989$ ) and were 0.8880 .8650 .840 and 0.747 in the remainder. An exponential model improved the correlation $(r>0.9)$ in the latter three subjects. For high intensity exercise linear $r$ values exceeded 0.9 in all subjects $(0.919-0.989$ for total high intensity exercise, $0.915-0.994$ for the initial burst). The slope of increase of dyspnoea with ventilation was nearly identical (table 2) when progressive and high intensity exercise were compared; it was somewhat smaller, though not significantly $(p=0.09)$ when initial high intensity exercise was compared with progressive exercise. The intercept with the ventilation axis was very similar, around 9-10 1/min, for all exercise protocols. A moderate but significant negative relationship were found between the $\mathrm{D} / \mathrm{V}_{\mathrm{E}}$ slope and $\mathrm{FEV}_{1}$ (fig 3): $\mathrm{D} / \dot{\mathrm{V}}$ slope $=5.26-2.34 \times \mathrm{FEV}_{1}(\mathrm{r}=$ $-0.583 ; p<0.01$; high intensity exercise).

\section{DYSPNOEA RATINGS: FIRST DAY VERSUS SECOND} DAY

Nine subjects performed progressive exercise on the first day and 10 subjects started with high intensity exercise. When the order effect of the test was examined, there was no significant difference between the first and the second study day for $\operatorname{Dmax}(92 \cdot 1 \%(2.6 \%) v$ $90.7 \%(2 \cdot 4 \%))$ or for $\mathrm{D} / \dot{\mathrm{V} E}$ slope $(2.90 \%$ $(0.20 \%)$ v $\left.2.83 \%(0.25 \%) 1^{-1} \mathrm{~min}\right)$.

REPRODUCIBILITY AT TWO MONTH INTERVAL Differences in HRmax, $\dot{V}$ Emax, $\dot{V O}_{2} \max , \mathrm{Dmax}$ and $\mathrm{D} / \dot{\mathrm{V}} \mathrm{E}$ slope between the baseline and the two month sessions were small and non-significant for both progressive and high intensity exercise. Individual data for VEmax, Dmax and $\mathrm{D} / \dot{\mathrm{V} E}$ slope are shown for progressive and initial high intensity exercise in figure 4.
Figure 4 Reproducibility of maximum ventilation ( $\dot{V}_{\text {Emax }}$ ), maximum dyspnoea (Dmax), and dyspnoea/ventilation slope (D/VE slope) in six patients with severe chronic obstructive lung disease studied twice at an interval of two months, during progressive and initial high intensity exercise. For each variable mean (SE) values of the baseline and of the two month session are joined by a thick line. 
Table 3 Reproducibility after two month interval: within subject coefficients of variation (median (range) values)

\begin{tabular}{|c|c|c|c|c|c|}
\hline & $H R \max$ & $\dot{V}_{E m a x}$ & $\dot{V} \mathrm{O}_{2} \max$ & $D \max$ & $D / \dot{V}_{E}$ slope \\
\hline $\begin{array}{l}\text { Progressive } \\
\text { Initial high intensity } \\
\text { Repeat high intensity } \\
\text { Whole high intensity }\end{array}$ & $\begin{array}{l}1 \cdot 8(0-7 \cdot 1) \\
3 \cdot 2(0-8 \cdot 0) \\
4 \cdot 4(0-7 \cdot 7) \\
\end{array}$ & $\begin{array}{l}7 \cdot 2(5 \cdot 1-9 \cdot 4) \\
5 \cdot 8(1 \cdot 9-6 \cdot 3) \\
4 \cdot 8(1 \cdot 8-10 \cdot 6) \\
\end{array}$ & $\begin{array}{l}6 \cdot 2(3 \cdot 6-7 \cdot 2) \\
4 \cdot 7(2 \cdot 4-13 \cdot 3) \\
5 \cdot 6(2 \cdot 2-8 \cdot 2) \\
\end{array}$ & $\begin{array}{l}4 \cdot 7(0-15 \cdot 7) \\
4 \cdot 6(0-8 \cdot 3) \\
8 \cdot 1(0-16 \cdot 6) \\
\end{array}$ & $\begin{array}{l}8.9(1 \cdot 2-28 \cdot 4) \\
4.9(1 \cdot 1-20.9) \\
\frac{6.0}{}(1.9-29.1)\end{array}$ \\
\hline
\end{tabular}

Abbreviations as in table 1.

Median within subject coefficients of variation are shown in table 3. Comparison of the variability of the same index between exercise protocols yielded insignificant differences. When the variability between the different indices was compared within each protocol, the only significant difference was that VEmax was less reproducible than $H R \max (p<0.01)$ during progressive exercise. Similarly, the reproducibility of the $D / \dot{V} E$ slope tended $(p=0.06)$ to be not so good as that of HRmax during progressive exercise. This tendency, however, was due exclusively to the presence in the group of one individual who exhibited a coefficient of variation of $28.4 \%$ (range $1 \cdot 2-12 \cdot 0 \%$ in the other subjects).

\section{Discussion}

Magnitude rating on a visual analogue scale has been used in many studies of exercise induced dyspnoea in normal subjects ${ }^{12817}$ and patients with chronic obstructive lung disease..$^{34-13}$ In all these studies exercise testing was performed conventionally as an incremental progressive test. This conflicts with the usual recommendation that in any psychophysical experiment sensory stimuli should be applied in random order to avoid artefacts. ${ }^{18}$ During a progressive exercise test subjects may be aware of the increasing work intensity and of the immediately preceding dyspnoea score, and hence may be cued to attach a higher sensation number to the next load increment. After considerable preliminary trials we devised a testing procedure consisting of two periods of low level exercise interspersed with two periods of high intensity exercise; the total exercise time, the maximum work load, and the cumulative work during the test were the same as in the progressive protocol used for comparison. Using this procedure we did not observe any arrhythmias or ischaemic changes on the electrocardiogram during high intensity exercise, which we ascribe to the beneficial effect of the preceding 10 watt warm up period. ${ }^{19}$ In this group of 19 subjects with chronic obstructive lung disease the dyspnoeaventilation slopes were very similar, whatever the temporal profile of the load. The mean rating of dyspnoea increased by about $29 \%$ of the length of the visual analogue scale every time the ventilation increased by $101 \mathrm{~min}^{-1}$ during both progressive and high intensity exercise.

The presence or absence of a point of reference is likely to have a strong influence on the sensitivity and the reproducibility of the assessment of dyspnoea. The visual analogue scale has been compared recently with the Borg scale, another widely used scale for assessing dyspnoea, which includes a descriptive framework of adjectives that is thought to allow subjects a point of reference. ${ }^{20}$ In a small group of six patients with chronic obstructive lung disease the visual analogue scale was used over a wider range than the Borg scale and was almost twice as sensitive. ${ }^{13}$ As some patients anchored solely with reference to everyday experience restrict the use of the visual analogue scale to its lower part, ${ }^{4}$ we anchored the upper end of the scale on a preliminary maximum exercise test in an attempt to persuade the subjects to use the whole range of the scale. Stark et al did not anchor the upper end of the scale in patients with respiratory disorders because the fatigue resulting from the anchoring test might impair exercise performance during the subsequent tests. ${ }^{421}$ We have circumvented this problem by allowing 24 hours to elapse between anchoring and the first session of dyspnoea rating. With this procedure only one subject from an initial sample of 21 was unable to use the point of reference given in the preliminary test and restricted the use of the visual analogue scale to its lower part.

In the present study we found, as have other investigators using the visual analogue scale or the Borg scale, ${ }^{1322}$ that the relation between dyspnoea and ventilation is linear during progressive exercise in most patients with chronic obstructive lung disease. Two subjects showed a plateauing of perceived dyspnoea despite a further increase in ventilation. This could be an artefact induced by the anchoring procedure, the same $100 \%$ level of breathlessness being experienced on the study day at a slightly lower load level than on the anchoring day, possibly because a mouthpiece was used. ${ }^{23}$ Alternatively, it could be an artefact due to the fact that the visual analogue scale is a closed scale. ${ }^{20}$ This is supported by the fact that other investigators have experienced this problem with the Borg scale, ${ }^{13224}$ and have allowed patients to select a number above the maximum on the Borg scale when breathlessness increased beyond what they had rated as maximal during progressive exercise. Interestingly, the plateauing of dyspnoea at high levels of ventilation was not observed in the two subjects when they were evaluated with the high intensity protocol.

We found a large intersubject variation in the rate of increase of dyspnoea with ventilation (see fig 3), which confirms that comparisons and therapeutic evaluations with this method should always be made on a within subject basis. ${ }^{21}$ The variability in $\mathrm{FEV}_{1}$ accounted for $34 \%$ of the variability of the $\mathrm{D} / \dot{\mathrm{V}} \mathrm{E}$ slope. The slope of the initial high intensity phase, though 
slightly less, was not significantly different from that seen with the progressive exercise or throughout the high intensity protocol. This finding suggests that a short, easily repeatable, single phase high intensity protocol could be useful in studies aimed at detecting an acute pharmacological effect on dyspnoea during exercise.

Reproducibility of dyspnoea rating has been studied in patients with chronic obstructive lung disease after intervals of one hour and one day $^{22}$ and at one to 10 day intervals, ${ }^{13}$ but not over longer periods. We studied six patients with chronic pulmonary obstructive disease after a two month interval. HRmax had the lowest within subject variability, a finding in agreement with the results of other studies ${ }^{1625}$; the variability of Dmax and of the $D / \dot{V} E$ slope compared well with that of D/VEmax and $\mathrm{V}_{2} \max$, though one individual showed poor reproducibility in visual analogue scale ratings. The finding that the $\mathrm{D} / \dot{\mathrm{V}} \mathrm{E}$ slope was generally reproducible after an interval of two months is useful as this interval is convenient for evaluating rehabilitation programmes.

In summary, we have found in 19 patients with chronic obstructive lung disease that the rate of increase of dyspnoea with increasing ventilation is the same whether measured during high intensity exercise or during conventional progressive exercise. No information was given to the patients about the temporal profile of the load during the exercise tests. The rate of increase was calculated on data that included the repeat as well as the initial burst of high intensity exercise. The sequence of exercise protocols was randomised. Our results strongly suggest that the visual analogue scale provides a valid assessment of dyspnoea in patients with respiratory disease during progressive exercise despite the theoretical objection that the patient may be aware that the work load will increase. The high intensity protocol might have an advantage in avoiding a plateauing artefact of dyspnoea ratings encountered in some patients at the end of the progressive test.

We thank Dr T Prigogine for permission to study one of his patients and Dr M Telerman for his support.

1 Stark RD, Gambles SA, Lewis JA. Methods to assess breathlessness in healthy subjects: a critical evaluation and application to analyze the acute effects of diazepam and promethazine on breathlessness induced by exercise or by exposure to raised levels of carbon dioxide. Clin $\mathrm{Sc}$ 1981;61:429-39.

2 Adams L, Chronos N, Lane R, Guz A. The measurement of breathlessness induced in normal subjects: validity of two scaling techniques. Clin Sci 1985;69:7-16.

3 Woodcock AA, Gross E, Geddes DM. Drug treatment of breathlessness: contrasting effects of diazepam and promethazine in pink puffers. $B M J 1981 ; 283: 343-6$.

4 Stark RD, Gambles SA, Chatterjee SS. An exercise test to assess clinical dyspnoea: estimation of reproducibility and sensitivity. Br J Dis Chest 1982;76:269-78.

5 Adams L, Lane $R$, Shea SA, Cockcroft A, Guz A. Breathlessness during different forms of ventilatory stimulation: a study of mechanisms in normal subjects and respiratory patients. Clin Sci 1985;69:663-72.

6 Lane R, Cockcroft A, Guz A. Voluntary isocapnic hyperventilation and breathlessness during exercise in normal subjects. Clin Sci 1987;73:519-23.

7 Freedman S, Lane R, Guz A. Breathlessness and respiratory mechanics during reflex or voluntary hyperventilation in patients with chronic airflow limitation. Clin Sci 1987;73:311-8.

8 Wilson RC, Jones PW. Influence of prior ventilatory experience on the estimation of breathlessness during exercise. Clin Sci 1990;78:149-53.

9 O'Neill PA, Stark RD, Allen SC, Stretton TB. The relationship between breathlessness and ventilation during steady-state exercise. Bull Eur Physiopathol Respir 1986;22:247-50.

10 Woodcock AA, Gross ER, Gellert A, Shah S, Johnson M, Geddes DM. Effects of dihydrocodeine, alcohol and caffeine on breathlessness and exercise tolerance in patients with chronic obstructive lung disease and normal blood gases. N Engl J Med 1981;305:1611-6.

11 Swinburn CR, Wakefield JM, Jones PW. Relationship between ventilation and breathlessness during exercise in chronic obstructive airways disease is not altered by prevention of hypoxaemia. Clin Sci 1984;67:515-9.

12 McKeon JL, Murree-Allen K, Saunders NA. Effects of breathing supplemental oxygen before progressive exercise in patients with chronic obstructive lung disease. Thorax 1988;43:53-6.

13 Muza SR, Silverman MT, Gilmore GC, Hellerstein HK, Kelsen SG. Comparison of scales used to quantitate the sense of effort to breathe in patients with chronic obstructive pulmonary disease. Am Rev Respir Dis 1990;141: 909-13.

14 Nicklaus TM, Burgin WW, Taylor JR Jr. Spirometric tests to diagnose suspected asthma Am Rev Respir Dis 1969;100:153-9.

15 Quanjer $\mathrm{Ph} \mathrm{H}$, ed. Standardized lung function testing. Bull Eur Physiopathol Respir 1983;19:suppl 5.

16 Noseda A, Carpiaux JP, Prigogine T, Schmerber J. Lung function, maximum and submaximum exercise testing in COPD patients: Reproducibility over a long interval. Lung 1989;167:247-57.

17 Wilson CR, Jones PW. A comparison of the visual analogue scale and modified Borg scale for the measurement of dyspnea during exercise. Clin Sci 1989;76:277-82.

18 Stevens SS. Issues in psychophysical measurement. Psychol Rev 1971;78:426-50.

19 Barnard RJ, Gardner GW, Diaco NV, McAlpin RN, Kattus AA. Cardiovascular responses to sudden strenuous exercise-heart rate, blood pressure and ECG. J Appl Physiol 1973;34:833-7.

20 Killian KJ. The objective measurement of breathlessness. Chest 1985;88 (suppl):84-90S.

21 Stark RD. Dyspnoea: Assessment and pharmacologica manipulation. Eur Respir J 1988;1:280-7.

22 Silverman M, Barry J, Hellerstein H, Janos J, Kelsen S Variability of the perceived sense of effort in breathing during exercise in patients with chronic obstructive pulduring exercise in patients with chronic obstructive

23 Simon PM, Basner RC, Weinberger SE, Fencl V, Weiss JW, Schwartzstein RM. Oral mucosal stimulation modulates intensity of breathlessness induced in normal subjects. $A m$ Rev Respir Dis 1991;144:419-22.

24 El-Manshawi A, Killian KJ, Summers E, Jones NL. Breath lessness during exercise with and without resistive loading. J Appl Physiol 1986;61:896-905.

25 Owens MW, Kinasewitz GT, Strain DS. Evaluating the effects of chronic therapy in patients with irreversible airflow obstruction. Am Rev Respir Dis 1986;134:935-7. 